\title{
Stereoelectroencephalography in children: a review
}

\author{
*Allen L. Ho, MD, ${ }^{1}$ Austin Y. Feng, BS, ${ }^{1}$ Lily H. Kim, BA, ${ }^{1}$ Arjun V. Pendharkar, MD, ${ }^{1}$ \\ Eric S. Sussman, MD, ${ }^{1}$ Casey H. Halpern, MD, ${ }^{1}$ and Gerald A. Grant, MD ${ }^{1,2}$

\begin{abstract}
'Department of Neurosurgery, Stanford University School of Medicine; and 2Division of Pediatric Neurosurgery, Lucile Packard Children's Hospital, Stanford, California
\end{abstract}

\begin{abstract}
Stereoelectroencephalography (SEEG) is an intracranial diagnostic measure that has grown in popularity in the United States as outcomes data have demonstrated its benefits and safety. The main uses of SEEG include 1) exploration of deep cortical/sulcal structures; 2) bilateral recordings; and 3) 3D mapping of epileptogenic zones. While SEEG has gradually been accepted for treatment in adults, there is less consensus on its utility in children. In this literature review, the authors seek to describe the current state of SEEG with a focus on the more recent technology-enabled surgical techniques and demonstrate its efficacy in the pediatric epilepsy population.
\end{abstract}

https://thejns.org/doi/abs/10.3171/2018.6.FOCUS18226

KEYWORDS stereoelectroencephalography; pediatric epilepsy; pediatric SEEG; frame-based SEEG; frameless SEEG; robot SEEG

$\mathrm{F}$ OR drug-resistant focal epilepsies, resective surgery is often the most effective treatment..$^{33}$ However, patient selection for epilepsy surgery relies on a multidisciplinary approach to epilepsy workup for proper identification of the epileptogenic zone (EZ). For many patients, noninvasive diagnostic measures can be sufficient for EZ localization, but many patients will require additional invasive monitoring. ${ }^{8}$ Stereoelectroencephalography (SEEG) is an intracranial diagnostic measure that has grown in popularity in the United States as outcomes data have demonstrated its benefits and safety. ${ }^{8}$ SEEG was first developed by Talairach and Bancaud in France during the 1950s as part of an integrative approach to epilepsy treatment. ${ }^{23}$ This method involves intracranial insertion of multilead depth electrodes into cortical and subcortical areas of interest. Electrographic data of focal seizures gathered from SEEG monitoring combined with clinical observations could more robustly identify seizure onset zones to target for resection. Current practice still follows this methodology, as SEEG implantation hypotheses require the synthesis of various multimodal diagnostic sources. ${ }^{8,15,23}$

Until recently, SEEG usage was not very common out- side of France, Italy, and Canada. Possible explanations for this trend are the lack of SEEG literature in the English language and/or the technical demands and learning curve associated with this technique. ${ }^{8}$ However, with the advent of newer technologies, such as improved imaging modalities, stereotactic image guidance, and robot-assisted navigation, SEEG is now gaining wide acceptance globally as the barriers to its adoption decrease. ${ }^{8}$ The main uses of SEEG include 1) exploration of deep cortical/sulcal structures; 2) bilateral recordings; and 3) 3D mapping of EZs. Prior to any invasive monitoring, a full multidisciplinary diagnostic workup should be completed to ensure that indications are sound for further monitoring. These indications generally revolve around discrepancies between noninvasive diagnostics, and they can be summarized as follows: ${ }^{3-5,7-10}$ 1) ictal or interictal electroencephalography (EEG) findings discordant with clinical semiology; 2) absence of cortical lesions on MRI despite convincing EEG seizure localization; 3) incongruence between abnormalities seen on MRI and EEG and clinical semiology localization, suggesting extralesional involvement; 4) focal abnormality seen on MRI discordant with electroclinical hypothesis; 5) hypothesized EZ involved with or close to

ABBREVIATIONS EEG = electroencephalography; $E Z$ = epileptogenic zone; SEEG = stereo-EEG.

SUBMITTED May 1, 2018. ACCEPTED June 28, 2018.

INCLUDE WHEN CITING DOI: 10.3171/2018.6.FOCUS18226.

${ }^{*}$ A.L.H. and A.Y.F. contributed equally to this work. 
eloquent areas; and 6) large, hemispheric, and/or multifocal abnormalities revealed by MRI that are discordant with localized ictal onset.

Beyond its diagnostic capabilities, SEEG can also be coupled with radiofrequency thermocoagulation, further broadening its utility. ${ }^{23}$ While SEEG has gradually been accepted for treatment in adults, there is less consensus on its utility in children. Our literature review seeks to describe the current state of SEEG with a focus on more recent technology-enabled surgical techniques and demonstrate its efficacy for the pediatric epilepsy population.

\section{Medically Refractory Epilepsy in Children}

Key differences in medically intractable epilepsy exist between children and adults. For instance, epileptogenic location often presents in different anatomical regions. Temporal lobe epilepsy is most common in adults, whereas many surgical series dealing with pediatric epilepsy have found that the EZs are frequently extratemporal (e.g., frontal or insular cortex) or multilobar., ${ }^{9,20}$ From a treatment standpoint, extratemporal and multilobar onsets in children are more likely to require invasive recordings due to their complexity compared with temporal seizures. Ictal semiology and scalp EEG alone have been shown to be far less effective in EZ localization in children. ${ }^{43}$ Additionally, identification of cortical dysplasias can be difficult on MRI due to incomplete myelination in children. ${ }^{12,43} \mathrm{Neu}-$ rological development is incomplete for younger patients, which can sometimes confound noninvasive workup that is often cognition, language, or task based. These differences suggest that pediatric patients may be particularly indicated for invasive recordings.

Despite these inherent difficulties, resection can still be beneficial for pediatric patients. The literature demonstrates that early surgery in appropriate cases can help improve brain development, as the harmful effects of the antiepileptic medications and seizures are minimized. ${ }^{12}$ Pediatric patients also have a distinct advantage over adults due to the plasticity of the brain, which may lend a neuroprotective factor against seizures or potential iatrogenic injury from epilepsy surgery. ${ }^{12}$

\section{Depth Versus Subdural Surface Recording}

Precise localization and characterization of the EZ is critical to achieve the goals of epilepsy surgery. Furthermore, investigation of the EZ needs to be hypothesis driven, based on seizure semiology, and supported by findings from clinical workup rather than a fishing expedition. The most popular technique for intracranial recording has been utilization of subdural grid and strip surface electrodes, where a craniotomy or burr hole is required for implantation. ${ }^{23,27,32}$ The advantage of subdural electrodes is the large surface area coverage of the cortex, providing excellent spatial resolution of superficial layers. However, the inability to record from deeper cortical and subcortical regions, the need for craniotomy, and the difficulty of comprehensive bilateral monitoring hamper its effectiveness. Selection criteria for SEEG specifically address these limitations: ${ }^{8-10}$ 1) potential for the EZ to be located in nonsuperficial regions, such as sulci and deep cortical areas (mesial temporal lobe, cingulate gyrus, insula, and posterior orbitofrontal areas); 2) need for bihemispheric coverage; 3) assessment of possible eloquent area involvement after clinical evaluation in the context of nonlesional MRI; and 4) reevaluation of patients who have undergone unsuccessful subdural electrode localization.

Despite similar goals, subdural electrodes and SEEG are indicated for relatively different seizure localizations. Subdural electrodes are well suited for EZ localization at the cortical surface or within eloquent cortex. SEEG utilizes electrodes that penetrate into brain parenchyma and is ideal for predicted subcortical or bilateral EZ involvement. In a rare direct comparison, a recent series by Yang et al. reported resective surgery outcomes following subdural electrode or SEEG evaluation conducted at the same institution..$^{52}$ The subdural $(n=52)$ and SEEG $(n=48)$ cohorts were selected according to a preoperative assessment that examined noninvasive diagnostics and the hypothesized EZ location. They found no significant differences in surgical sites (e.g., frontal lobe, multilobe), types (e.g., local cortex resection, single lobe resection), seizure control, cognitive outcomes, or pathological results (e.g., tumor, cortical dysplasia). SEEG was preferred when more than 3 lobes were involved or exclusively used when EZ involvement was probable in bilateral hemispheres. SEEG was also associated with a significantly lower rate of intracranial hemorrhage and surgical site infection than subdural electrodes $(4.2 \%$ vs $17.3 \%, \mathrm{p}<0.05)$. With appropriately selected patients, both methods can be effective at localizing the area of seizure onset ${ }^{52}$ (Tables 1 and 2).

SEEG is also valuable in the reevaluation of patients with inconclusive findings on subdural EEG monitoring and vice versa since these techniques are complementary. In a short series examining this subset of patients, SEEG helped localize the EZ in more than $90 \%$ of cases (13/14). Seizure freedom was achieved in $60 \%$ of patients who underwent resection. ${ }^{46}$ For most patients in whom subdural electrode evaluation yielded nonlocalizable ictal patterns, ictal onset was ultimately found in deep cortical areas (e.g., insula and posterior cingulate cortex); thus, further subdural investigation may not have been helpful and risked greater surgical morbidity. Although SEEG ultimately led to identification of the EZ, the data gained with initial subdural electrode recordings should not be discounted. SEEG must be guided by a personalized implantation strategy, which the information provided by prior subdural grid recording can facilitate. ${ }^{46}$

Proper usage and understanding of surface versus depth electrodes can create synergy in EZ localization and lead to improved outcomes. A recent series from the Cleveland Clinic comparing subdural electrodes and SEEG outcomes reported EZ localization at $77 \%$ and $97 \%$, respectively, with similar rates of seizure freedom after resection (68\% and 70\%). ${ }^{33}$ Coming from an institution well versed in both techniques, these rates are higher than those reported by other centers, where seizure-freedom rates range between $30 \%$ and $64 \%$ (Table 1). That study also included a substantial cohort of pediatric patients (31/102), suggesting that the enhanced EZ localization through SEEG availability may benefit both adult and pediatric populations. With greater experience and more 
TABLE 1. Literature review of pediatric SEEG series*

\begin{tabular}{|c|c|c|c|c|c|}
\hline Authors \& Year & Modality & No. of Pts & EZ Localization & Sz Freedom & Complications \\
\hline Cossu et al., 2012 & Frame & 15 & $92.9 \%$ & $60 \%$ & $0 \%$ \\
\hline Gonzalez-Martinez et al., 2013 & Frame & 100 (17 pediatric pts) & $96 \%$ & $62.3 \%$ & $3 \%$ \\
\hline Gonzalez-Martinez et al., $2014^{18}$ & Frame & 122 (21 pediatric pts) & $94 \%$ & $62 \%$ & $2.5 \%$ \\
\hline Taussig et al., 2014 & Robot & 65 & NR & $36.4 \%$ & $0 \%$ \\
\hline Gonzalez-Martinez et al., $2014^{17}$ & Frame & 30 & $86.7 \%$ & $55.5 \%$ & $3 \%$ \\
\hline González-Martínez et al., 2016 & Robot & 68 (14 pediatric pts) & NR & $64.29 \%$ & $0 \%$ \\
\hline De Benedictis et al., 2017 & Robot & 36 & NR & NR & $0 \%$ \\
\hline Miller et al., 2017 & Robot & 11 & NR & $36.4 \%$ & $0 \%$ \\
\hline van der Loo et al., 2017 & Frame & 71 (17 pediatric pts) & NR & NR & $11.8 \%$ \\
\hline Budke et al., 2017 & Frameless & 15 & $100 \%$ & NR & $0 \%$ \\
\hline \multirow[t]{2}{*}{ Abel et al., 2018} & Robot & 17 & NR & $33.33 \%$ & $23.5 \%$ \\
\hline & Frame & 18 & NR & $41.67 \%$ & $11.1 \%$ \\
\hline
\end{tabular}

$\mathrm{NR}=$ not reported; pts = patients; $\mathrm{Sz}=$ seizure.

* Includes studies from the last decade.

precise guidelines for SEEG or subdural electrode evaluations, patients' seizures can be optimally characterized and treated.

\section{Safety}

Despite invasion of the brain parenchyma with depth electrode placement, the safety profile of SEEG compares favorably to that of subdural electrodes. Total complication rates (e.g., hemorrhage, infection, psychiatric) are below $16 \% .{ }^{28}$ Meta-analyses of published studies have calculated aggregate risks of intracranial hemorrhage and surgical site infection with subdural electrode placement at $4 \%$ and $2.3 \%$, and with SEEG at $1 \%$ and $0.8 \%$, respectively. ${ }^{23,32}$ Total mortality rates range between $0 \%$ and $1.4 \% .^{14}$

SEEG complications in adult patients have been studied extensively. One of the largest series by Talairach et al. reported an intracranial hemorrhage rate of $0.5 \%$ in 560 patients. ${ }^{43}$ A series by Tanriverdi et al. evaluated 491 patients with SEEG performed at a single institution and by a single surgeon. The authors reported a total complication rate of $7.1 \%$, with no deaths or major complications.
Interestingly, monitoring duration was found to have no significant effect on complication rate. ${ }^{44}$ A series by Mathon et al. of 163 intracranial procedures reported an overall complication rate of $4.9 \%$, with no deaths or permanent morbidity. ${ }^{28}$ Patients with nonlesional epilepsy were found to be at a significantly higher risk of hemorrhagic complications, but this association was uncorroborated by other studies. Certain techniques are common between these large series. Both Tanriverdi and Mathon and their colleagues reported the use of twist drills instead of burr holes. While burr holes may allow visualization of cortical vessels, the smaller twist-drill hole may have helped reduce hemorrhage rates. Additionally, both authors emphasized the importance of sterile technique to prevent infection. Mathon et al. reported that from 2007 to 2014, infections in patients dropped to 0 once their postoperative management of head bandages switched from changing every 2 days to keeping the same bandage for the duration of monitoring.

For children, the few published series for SEEG have reported minimal adverse events (Table 3). One series of 35 pediatric patients reported 1 electrode breakage but

TABLE 2. Literature review of pediatric invasive EEG (grid, strips, and depth) series*

\begin{tabular}{lcccc}
\hline \multicolumn{1}{c}{ Authors \& Year } & No. of Pts & EZ Localization & Sz Freedom & Complications \\
\hline Simon et al., 2003 & 64 & NR & NR & $32.8 \%$ \\
\hline Önal et al., 2003 & 35 & NR & $40 \%$ & $57.1 \%$ \\
\hline Bauman et al., 2005 & 15 & NR & $60 \%$ & NR \\
\hline Musleh et al., 2006 & 112 & NR & NR & $13.1 \%$ \\
\hline Johnston et al., 2006 & 29 & NR & NR & $6.89 \%$ \\
\hline Van Gompel et al., 2008 & 189 (52 pediatric pts) & $79 \%$ & $47 \%$ & $6.6 \%$ \\
\hline Lee et al., 2008 & 92 (82 pediatric pts) & NR & NR & $16.7 \%$ \\
\hline Wong et al., 2009 & 71 (17 pediatric pts) & NR & $77.6 \%$ & $22.22 \%$ \\
\hline Blauwblomme et al., 2011 & 95 & $82.1 \%$ & $40.5 \%$ & $48.9 \%$ \\
\hline Kim et al., 2011 & 12 & $100 \%$ & $75 \%$ & $0 \%$ \\
\hline
\end{tabular}

\footnotetext{
* Includes studies from the last 15 years.
} 
TABLE 3. Literature review of surgical characteristics of pediatric SEEG series*

\begin{tabular}{|c|c|c|c|c|c|c|c|}
\hline Authors \& Year & Modality & $\begin{array}{l}\text { Entry Point } \\
\text { Error (mm) }\end{array}$ & $\begin{array}{l}\text { Target Point } \\
\text { Error (mm) }\end{array}$ & $\begin{array}{l}\text { OR Time } \\
\text { (mins) }\end{array}$ & Complications & $\begin{array}{l}\text { No. of } \\
\text { Leads }\end{array}$ & $\begin{array}{c}\text { Average } \\
\text { Leads per Pt }\end{array}$ \\
\hline Cossu et al., 2012 & Frame & NR & NR & NR & $0 \%$ & 181 & 12.1 \\
\hline Gonzalez-Martinez et al., 2013 & Frame† & NR & NR & NR & $3 \% ; 0.2 \%$ hemorrhage rate/electrode & 1310 & 13.1 \\
\hline $\begin{array}{l}\text { Gonzalez-Martinez et al., } \\
\text { 201418 }\end{array}$ & Frame† & NR & NR & 107 & $2.5 \% ; 0.18 \%$ hemorrhage rate/electrode & 1586 & 13 \\
\hline Taussig et al., 2014 & Robot & NR & NR & NR & $0 \%$ & 748 & 11.5 \\
\hline Gonzalez-Martinez et al., $2014^{17}$ & Frame & NR & NR & NR & $3 \% ; 0.2 \%$ hemorrhage rate/electrode & 402 & 13.4 \\
\hline González-Martínez et al., 2016 & Robot† & Median 1.2 & Median 1.7 & 130 & $4 \%$ (2 SDHs \& 2 IPHs) & 1245 & 12.5 \\
\hline De Benedictis et al., 2017 & Robot & Median 1.5 & Median 1.96 & 289.7 & $0 \%$ & 386 & 10.7 \\
\hline Miller et al., 2017 & Robot & NR & NR & 98 & $0 \%$ & 87 & 7.9 \\
\hline van der Loo et al., 2017 & Frame† & Median 1.54 & Median 2.93 & 135 & $\begin{array}{l}9.2 \% \text { minor complications; } 2.6 \% \text { major } \\
\text { complications }\end{array}$ & 902 & 12.7 \\
\hline Budke et al., 2017 & Frameless & Mean 3.64 & Mean 2.96 & 90 & $6.67 \%(1 \mathrm{SDH})$ & 111 & 7.4 \\
\hline \multirow[t]{2}{*}{ Abel et al., 2018} & Robot & NR & NR & 304 & $\begin{array}{l}23.5 \% \text { (5 SDHs, } 2 \text { IPHs, } 1 \text { focal edema, } \\
1 \text { transient paresthesia) }\end{array}$ & 265 & 15.6 \\
\hline & Frame & NR & NR & 352 & 11.1\% (1 SDH, $1 \mathrm{IPH}, 1 \mathrm{HA})$ & 264 & 14.7 \\
\hline
\end{tabular}

$\mathrm{HA}=$ headache; $\mathrm{IPH}=$ intraparenchymal hematoma; $\mathrm{SDH}=$ subdural hematoma.

* Includes studies from the last decade.

$\dagger$ Includes adult patients.

without neurological complications. ${ }^{11}$ In another series of 482 adult and pediatric patients, 1 pediatric patient died of brain edema and underlying electrolytic disturbances. ${ }^{6}$ In comparison, morbidities related to subdural electrodes in the pediatric population have been reported to be as high as $6 \%$ for infection, $14 \%$ for cerebral edema, and $25 \%$ for intracranial hemorrhage. ${ }^{4,10}$ While the long-term effects of SEEG implantation have yet to be studied, the scarcity of complications also suggests that pediatric patients can tolerate long-term monitoring, with reports of up to 3 weeks of recording. ${ }^{10}$ SEEG is particularly valuable for patients whose subdural electrode evaluation may have been unable to fully delineate the EZ, possibly leading to an ineffective resection. In this situation, additional subdural electrode evaluation is unable to probe deeper areas, and revision open craniotomy runs the risk of increased morbidity due to extensive scarring and neurovascular compromise. ${ }^{4,10}$ Particularly for children, the trauma of multiple open surgeries can also hamper their overall development. SEEG addresses both issues by providing recordings of deeper cortical regions and avoiding the need for repeat craniotomy. ${ }^{8-10}$

SEEG is not without its limitations. A relative contraindication for SEEG implantation is a bone thickness less than $2 \mathrm{~mm} \cdot{ }^{32}$ Retaining anchor bolts are needed for stable electrode placement, and sufficient bone thickness is important for adequate bolt fixation. However, the electrodes can be placed in patients with very thin bone and then secured without a bolt. Complete eloquent cortex mapping may be inadequate, even with thoughtful SEEG placement, such as in the dominant perisylvian or rolandic regions. Although 3D spatial precision is readily achieved, SEEG contacts are limited by electrode configurations toward the distal tip and do not always facilitate contiguous coverage. These limitations emphasize the importance of developing an EZ hypothesis supported by other noninvasive diagnostic data.

Advances in technology can also play a critical role in SEEG safety. Novel 3D digital subtraction angiography (DSA) used in 191 SEEG patients yielded only 2 minor intracerebral hemorrhages with no permanent sequelae? Compared with standard biplanar angiography, 3D DSA may aid in planning for the oblique trajectories by more modern arc-based stereotactic frames and stereotactic robots. Noninvasive modalities such as $\mathrm{CT}$ angiography (CTA) or double-dose gadolinium MR angiography (MRA) are also available and avoid the morbidity of DSA, with similar benefits of vessel avoidance during SEEG planning.?

\section{Different SEEG Techniques}

Different methodologies for SEEG have evolved with the development of new techniques and technologies (Table 1). These methods fall into 3 types: frame based, frameless, and robot assisted. Regardless of type, the fundamental principles of SEEG are relatively unchanged. All procedures first involve generating a preimplantation plan detailing electrode depths and trajectories, often facilitated with noninvasive measurements. The step is arguably the most important because the recording hinges on proper localization of the EZ and ictal network and avoidance of major cortical vessels to minimize hemorrhage risk. The skull and dura are penetrated, and guiding bolts are fixed to the entry point. Stylets may be inserted into the parenchyma to the desired target point and then withdrawn, or semirigid electrodes can be utilized that do not require this step. Finally, electrodes are advanced to their target points and secured to the guiding bolts. Postoperative imaging is usually performed to confirm elec- 

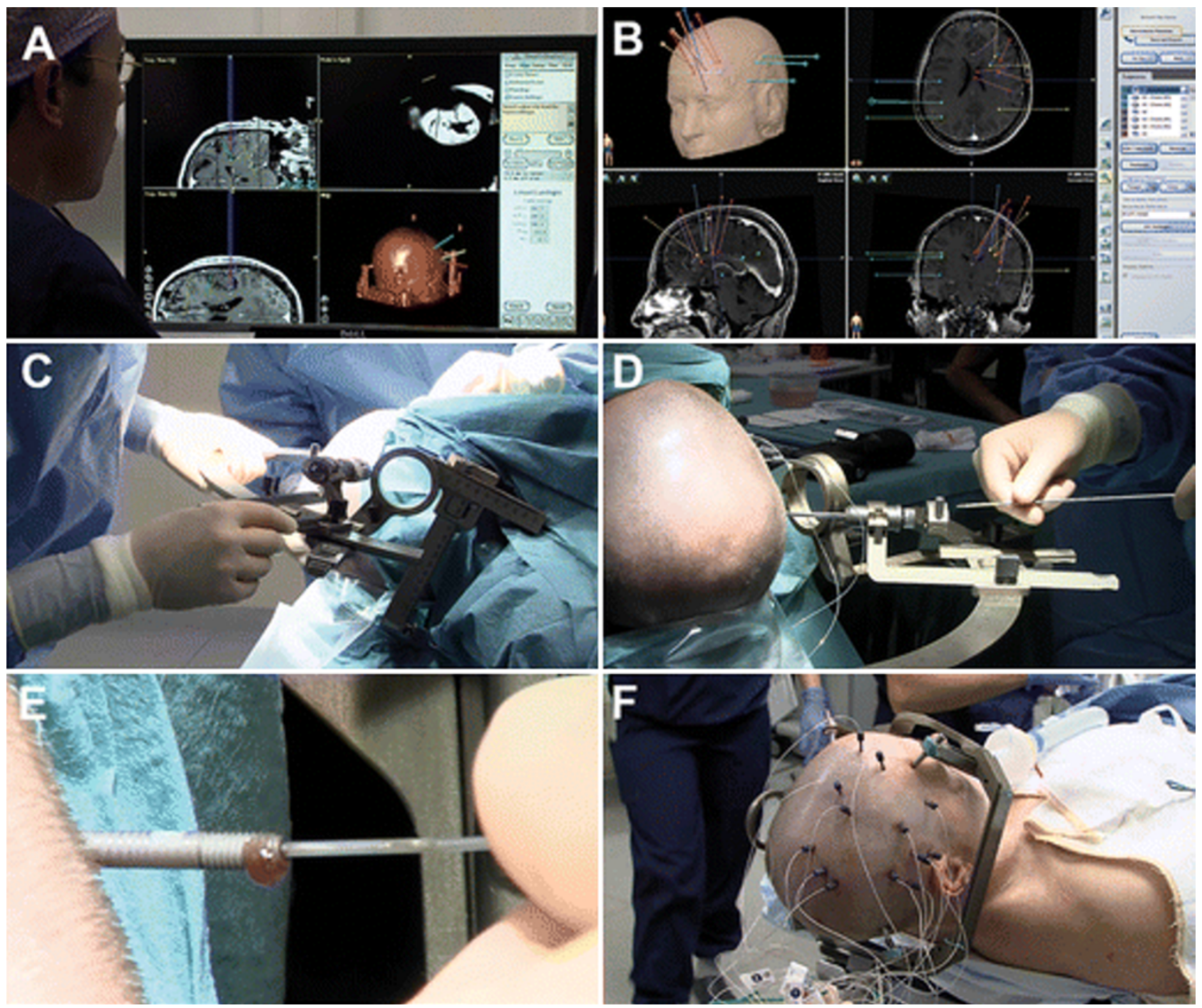

FIG. 1. Frame-based SEEG methodology. A and B: Electrode trajectories are planned using navigation software that fuses preoperative volumetric MRI and postframe CT scans and provides frame-based coordinates and electrode implantation depths. C: Coordinates of the Leksell frame are set by the operating neurosurgeon. D: The stylet is introduced through the guiding screw to a premeasured length to create the electrode track. E: Insertion of the depth electrode through the screw. F: Patient at the end of the procedure, after implantation of 13 depth electrodes and placement of fixation bolts. Reproduced from van der Loo et al: Acta Neurochir (Wien) 159:1733-1746, 2017. @2017 van der Loo LE, Schijns OEMG, Hoogland G, Colon AJ, Wagner GL, Dings JTA, Kubben PL. Published with permission by CC BY-NC 4.0 (https://creativecommons.org/licenses/by-nc/4.0/).

trode locations and rule out intracranial hemorrhage before the recording begins.

The original SEEG platform pioneered by Talairach and Bancaud was frame based (Fig. 1). The stereotactic frame allows for precise electrode placement by manipulating $\mathrm{x}, \mathrm{y}$, and $\mathrm{z}$ coordinates along its moveable arc. To utilize the frame, imaging is usually performed for registration after its installation on the patient. MRI and CT are common, but other forms, such as telemetric radiography and stereoscopic cerebral angiography, have also been utilized..$^{9,29,48,53}$ More recently, a myriad of stereotactic planning software has been developed (e.g., StealthStation, iPlan, Neurotech) to assist implantation. In a large series by Cossu et al. of 211 patients who underwent SEEG with the Talairach frame, $81 \%$ of patients could undergo resec- tion, resulting in $44 \%$ achieving seizure freedom with a 3\% SEEG-related morbidity rate. ${ }^{11}$ A smaller series by McGonigal et al. demonstrated rates of $96 \%$ for EZ localization, 53\% for postsurgery seizure freedom, and 3\% for complication. ${ }^{29}$ Traditional frames (e.g., Talairach, Leksell) attain good accuracy, indicated by a low entry point (EP) and target point (TP) error mean. ${ }^{6,19,48}$ A recent metaanalysis of accuracy of 3 frame-based SEEG studies determined an EP error mean of $1.43 \mathrm{~mm}$ (95\% CI 1.35-1.51 $\mathrm{mm})$ and TP error mean of $1.93 \mathrm{~mm}(95 \%$ CI 1.05-2.81 $\mathrm{mm}$ ), respectively. ${ }^{47}$ Newer customizable frames, such as StarFix, can achieve even greater accuracy, with an EP error mean of $0.68 \mathrm{~mm}$ and TP error mean of $1.64 \mathrm{~mm} .^{2}$ Despite the accuracy of the frame-based system, there are still limitations. The frame itself is both large and cum- 


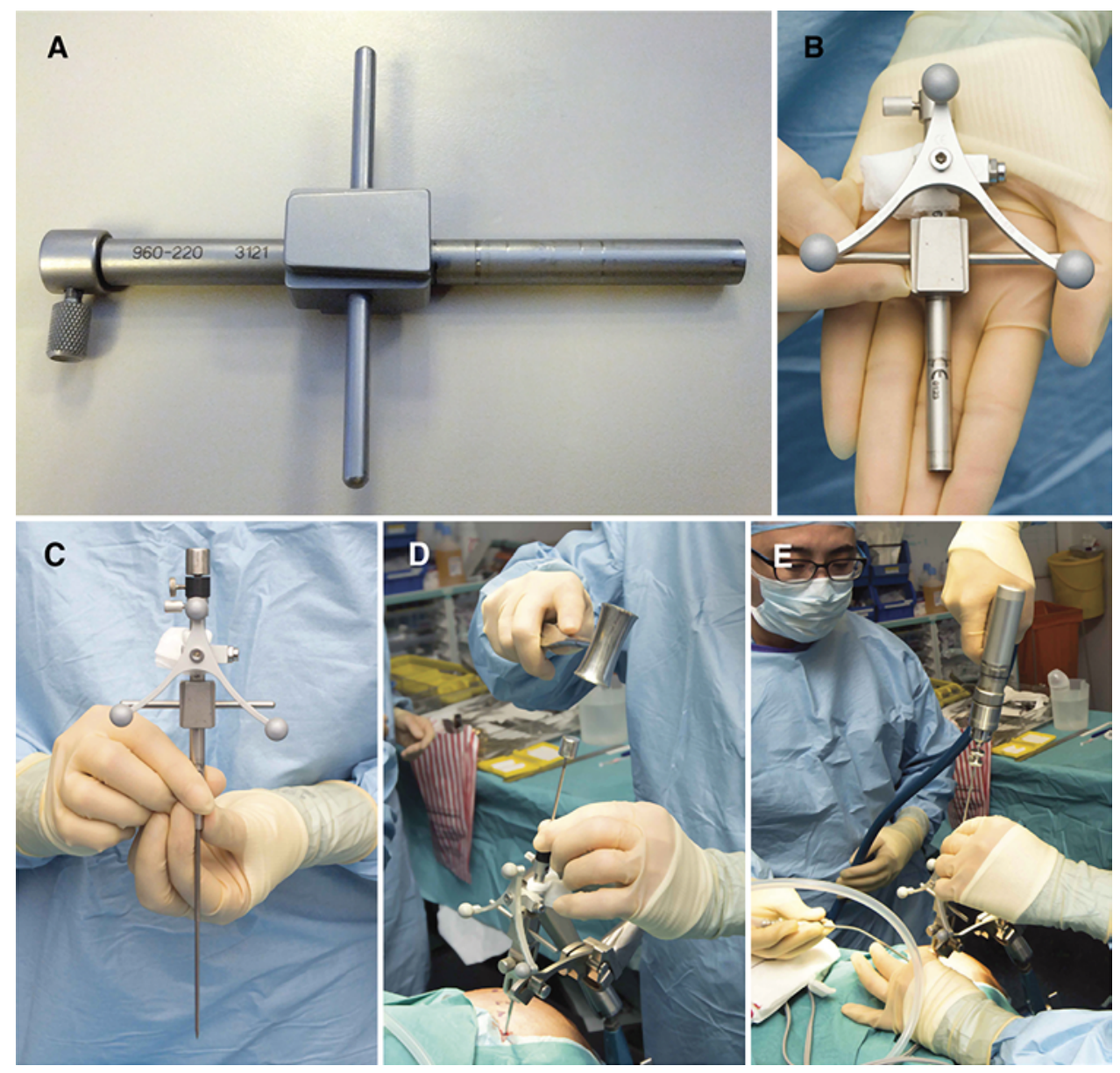

FIG. 2. Frameless SEEG methodology. A: Precision-aiming device. B: Precision-aiming device with the SureTrack image guidance device attached. C: Precision-aiming device and SureTrack device with the metal spike inserted. D: Delivery of hammered divot into the outer table of the skull under frameless stereotactic conditions. E: Drilling of the trajectory through the skull under frameless stereotactic conditions. Reproduced from Nowell M, Rodionov R, Diehl B, Wehner T, Zombori G, Kinghorn J, et al: A novel method for implementation of frameless StereoEEG in epilepsy surgery, 2014, Neurosurgery 10 (Supp/ 4):525-534, by permission of Congress of Neurological Surgeons.

bersome, which may lead to increased operative time as well as restrict potential electrode trajectories..$^{30,36}$ Installation in awake patients can be uncomfortable, as fixation requires pins to be screwed into the scalp. This may be especially difficult for pediatric patients who have smaller and more fragile skulls or those who cannot tolerate awake placement, lengthening the duration of general anesthesia for SEEG placement.

Frameless methods stabilize the head with a clamp and use neuronavigation platforms for electrode guidance (Fig. 2). Neuronavigation platforms integrate imaging information to help plan and execute safe electrode trajectories. Frameless systems utilize various methods, such as skullaffixed and arm-based devices, to attach to the head. Using imaging data, they can create virtual anatomical models to guide surgery. Different approaches also exist for registering the landmarks needed for the computer-generated model, from laser facial registration to skin fiducials to skull fiducials. Target point accuracy observed in multiple frameless SEEG series has ranged from 2.0 to 4.8 $\mathrm{mm}$, which compares less favorably with frame-based SEEG. ${ }^{30,34,36,50}$ This is supported by a meta-analysis of frameless accuracy, which determined an EP error mean of $2.45 \mathrm{~mm}(95 \%$ CI $0.39-4.51 \mathrm{~mm})$ and TP error mean of $2.89 \mathrm{~mm}(95 \%$ CI $2.34-3.44 \mathrm{~mm}){ }^{47}$ The accuracy of frameless systems can be hindered by patient motion during imaging and improper placement of registered markers. ${ }^{41}$ Hou et al. directly compared outcomes between Leksell frame-based and StealthStation (Medtronic S7 neuronavigation system) frameless SEEG. They found no significant difference in implantation error measurements $(2.03 \pm 0.98 \mathrm{~mm}$ vs $1.79 \pm 0.82 \mathrm{~mm})$ or EZ localization rates in their series $(88.9 \%$ vs $82.1 \%$ ) between frameless and frame-based SEEG. ${ }^{22}$ No hemorrhage or infections were reported. Looking at operative times per electrode, frameless times were significantly reduced compared with frame-based times (19.4 vs $34.5 \mathrm{~min} /$ electrode, respectively). ${ }^{22}$ It should be noted that this frameless implantation time is relatively high compared with those reported in the literature. Roessler et al. averaged 12 minutes for each electrode. ${ }^{38}$ Reporting on pediatric patients, Budke et al. averaged 15.7 min/electrode fixation. ${ }^{5}$ Very few complications were observed in the studies of frameless SEEG, although this may be due to fewer patients. ${ }^{29-31}$ Roessler et 

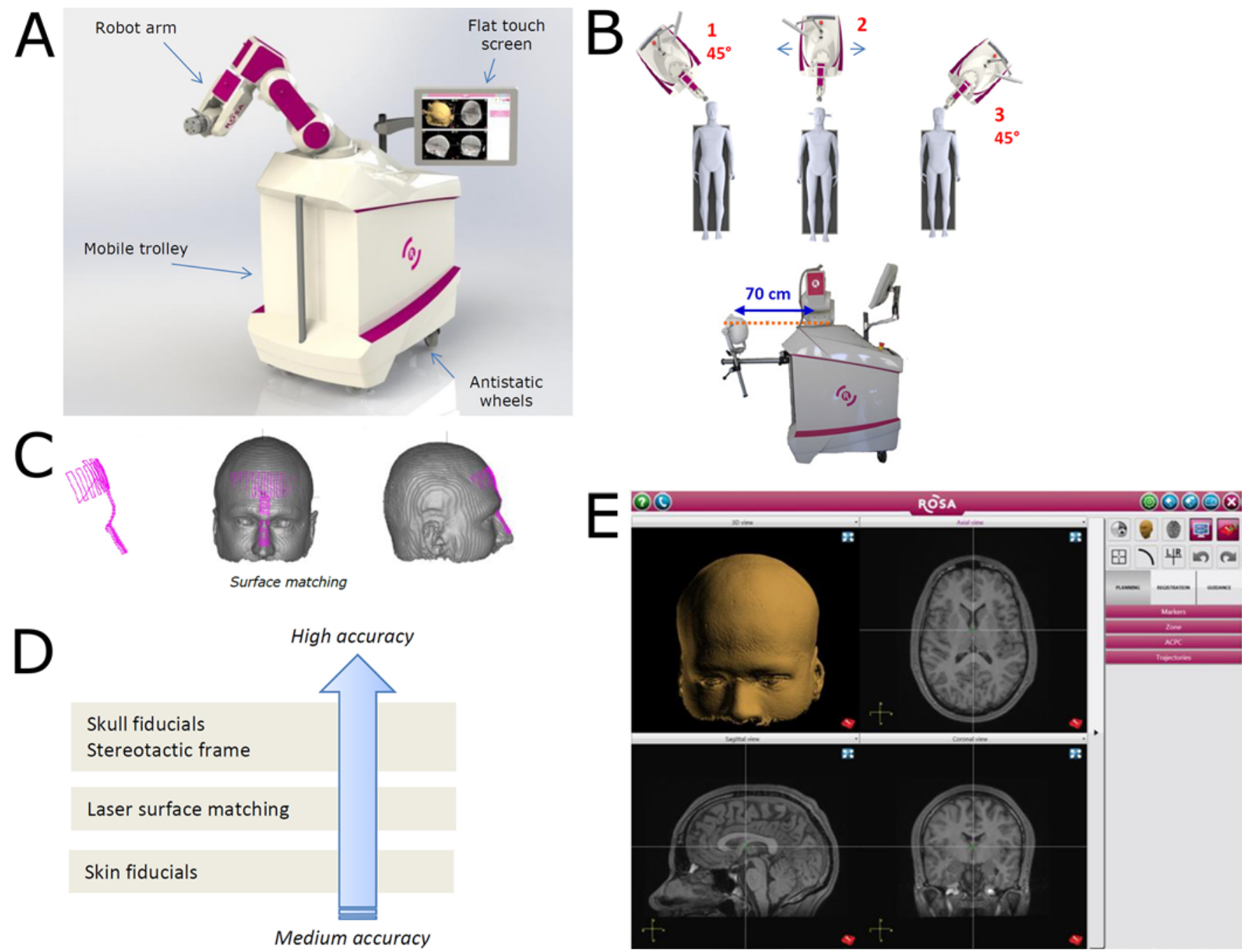

FIG. 3. Robot-assisted SEEG methodology. A: The ROSA robot consists of a robot arm with 6 degrees of freedom attached to a mobile floor-fixed base unit. B: The base unit attaches directly to the cranial head clamp, and platform positioning is critical and dependent on planned location of SEEG electrodes. C: The robot arm can be used for semiautomatic laser-based facial recognition, which is used to register the patient to the images. D: Accuracy of registration increases from skin-placed adhesive fiducials, to laser facial surface matching, to implanted skull fiducials and placement of the stereotactic frame. E: Working screen view of the native ROSA planning software that is utilized for image fusion and trajectory planning. Reproduced from Ho et al: Robot-guided pediatric stereoelectroencephalography: single-institution experience. J Neurosurg Pediatr [epub ahead of print August 17, 2018. DOI: 10.3171/2018.5.PEDS17718], with permission from AANS.

al. and Budke et al. both reported no significant complications with 6 and 15 patients, respectively. The advantages of frameless systems are well suited for pediatric patients. Reduced operative time decreases the duration of general anesthesia. Eliminating the bulky stereotactic frame improves both ease of use and operative trajectory options, both of which are beneficial as pediatric patients do not tolerate awake frame placement and often require more extensive electrode placement.

Both frame-based and frameless methods can be augmented with a robotic arm to assist with trajectory guidance (Fig. 3). The technical precision of robotic systems (e.g., ROSA, Neuromate) combined with neuronavigation software makes robot-assisted SEEG highly accurate and efficient. Cardinale et al. demonstrated improved accuracy of Neuromate-assisted (EP/TP error mean $=0.78 / 1.77$ $\mathrm{mm})$ over frame-based SEEG $(\mathrm{EP} / \mathrm{TP}$ error mean = $1.43 / 2.69 \mathrm{~mm}) .{ }^{6}$ González-Martínez et al. reported similar $\mathrm{EP} / \mathrm{TP}$ error mean values $(1.2 / 1.7 \mathrm{~mm})$ for ROSA-assisted SEEG. ${ }^{16}$ This study also reported a significant decrease in operative time between frame-based and robot-assisted SEEG (352 vs 130 minutes). Authors have reported major complication rates of $0 \%-1.2 \%$, suggesting comparable safety to non-robot-assisted techniques. ${ }^{6,16,21,39,42}$ Thus, the primary advantage of robot-assisted SEEG is this substantial decrease in operation time without sacrificing accuracy or safety. Several series of ROSA-assisted SEEG in pediatric populations have been reported. De Benedictis et al. reported on 36 SEEG procedures with a mean operative time of 204.3 minutes, an $89 \%$ rate of successful EZ identification, and no postoperative complications..$^{13}$ Miller et al. reported on 11 SEEG procedures with a mean 
operative time of 98 minutes, a $100 \%$ rate of successful EZ identification, and also no postoperative complications. ${ }^{31}$ Despite their small patient numbers, these studies indicate the exciting potential of robot-assisted SEEG for pediatric patients. However, as a relatively new technology, adoption of robot-assisted SEEG is hindered by cost, a learning curve, and the possibility of mechanical failure. Long-term data of postoperative robot-assisted SEEG also need to be analyzed to ensure safety and efficacy. Still, this technology holds much promise for the future of SEEG.

\section{Conclusions}

Stereoelectroencephalography is an effective diagnostic tool to incorporate into the workup of patients with medically refractory epilepsy. It is especially suitable for children for a variety of reasons, such as the propensity of extratemporal epileptogenic zones in children that warrant intracranial investigation and the ability to avoid large craniotomies with SEEG. Compared with the more commonly utilized subdural grids and strips, SEEG is superior in localizing deep, noncortical, or bilateral EZs. It also gives many patients who may have had unsuccessful cortical surface EEG a recording modality with more spatial resolution for identifying EZs. SEEG has demonstrated safety, with low rates of infection and hemorrhage. Given these advantages, SEEG has become a critical tool in the neurosurgeon's armamentarium for pediatric epilepsy surgery. What remains to be proven is whether SEEG improves our pediatric patients' outcomes and quality of life in a durable fashion over subdural grids. We challenge the reader to gather these data in a prospective fashion to achieve the best possible Engel outcomes and at the same time to minimize patient morbidity.

\section{References}

1. Abel TJ, Varela Osorio R, Amorim-Leite R, Mathieu F, Kahane P, Minotti L, et al: Frameless robot-assisted stereoelectroencephalography in children: technical aspects and comparison with Talairach frame technique. J Neurosurg 22:37-46, 2018

2. Balanescu B, Franklin R, Ciurea J, Mindruta I, Rasina A, Bobulescu RC, et al: A personalized stereotactic fixture for implantation of depth electrodes in stereoelectroencephalography. Stereotact Funct Neurosurg 92:117-125, 2014

3. Bauman JA, Feoli E, Romanelli P, Doyle WK, Devinsky O, Weiner HL: Multistage epilepsy surgery: safety, efficacy, and utility of a novel approach in pediatric extratemporal epilepsy. Neurosurgery 56:318-334, 2005

4. Blauwblomme T, Ternier J, Romero C, Pier KST, D'Argenzio L, Pressler R, et al: Adverse events occurring during invasive electroencephalogram recordings in children. Neurosurgery 69 (2 Suppl Operative):ons169-ons175, 2011

5. Budke M, Avecillas-Chasin JM, Villarejo F: Implantation of depth electrodes in children using VarioGuide ${ }^{\circledR}$ frameless navigation system: technical note. Oper Neurosurg (Hagerstown) [epub ahead of print], 2017

6. Cardinale F, Cossu M, Castana L, Casaceli G, Schiariti MP, Miserocchi A, et al: Stereoelectroencephalography: surgical methodology, safety, and stereotactic application accuracy in 500 procedures. Neurosurgery 72:353-366, 2013

7. Cardinale F, Pero G, Quilici L, Piano M, Colombo P, Moscato A, et al: Cerebral angiography for multimodal surgical planning in epilepsy surgery: description of a new three- dimensional technique and literature review. World Neurosurg 84:358-367, 2015

8. Chabardes S, Abel TJ, Cardinale F, Kahane P: Commentary: Understanding stereoelectroencephalography: what's next? Neurosurgery 82:E15-E16, 2018

9. Cossu M, Cardinale F, Castana L, Citterio A, Francione S, Tassi L, et al: Stereoelectroencephalography in the presurgical evaluation of focal epilepsy: a retrospective analysis of 215 procedures. Neurosurgery 57:706-718, 2005

10. Cossu M, Cardinale F, Castana L, Nobili L, Sartori I, Lo Russo G: Stereo-EEG in children. Childs Nerv Syst 22:766778, 2006

11. Cossu M, Cardinale F, Colombo N, Mai R, Nobili L, Sartori I, et al: Stereoelectroencephalography in the presurgical evaluation of children with drug-resistant focal epilepsy. J Neurosurg 103 (4 Suppl):333-343, 2005

12. Cossu M, Schiariti M, Francione S, Fuschillo D, Gozzo F, Nobili L, et al: Stereoelectroencephalography in the presurgical evaluation of focal epilepsy in infancy and early childhood. J Neurosurg Pediatr 9:290-300, 2012

13. De Benedictis A, Trezza A, Carai A, Genovese E, Procaccini E, Messina R, et al: Robot-assisted procedures in pediatric neurosurgery. Neurosurg Focus 42(5):E7, 2017

14. Derrey S, Lebas A, Parain D, Baray MG, Marguet C, Freger $\mathrm{P}$, et al: Delayed intracranial hematoma following stereoelectroencephalography for intractable epilepsy: case report. J Neurosurg Pediatr 10:525-528, 2012

15. Gonzalez-Martinez J, Bulacio J, Alexopoulos A, Jehi L, Bingaman W, Najm I: Stereoelectroencephalography in the "difficult to localize" refractory focal epilepsy: early experience from a North American epilepsy center. Epilepsia 54:323-330, 2013

16. González-Martínez J, Bulacio J, Thompson S, Gale J, Smithason S, Najm I, et al: Technique, results, and complications related to robot-assisted stereoelectroencephalography. Neurosurgery 78:169-180, 2016

17. Gonzalez-Martinez J, Lachhwani D: Stereoelectroencephalography in children with cortical dysplasia: technique and results. Childs Nerv Syst 30:1853-1857, 2014

18. Gonzalez-Martinez J, Mullin J, Bulacio J, Gupta A, Enatsu R, Najm I, et al: Stereoelectroencephalography in children and adolescents with difficult-to-localize refractory focal epilepsy. Neurosurgery 75:258-268, 2014

19. Gonzalez-Martinez J, Mullin J, Vadera S, Bulacio J, Hughes G, Jones S, et al: Stereotactic placement of depth electrodes in medically intractable epilepsy. J Neurosurg 120:639-644, 2014

20. Gonzalez-Martinez J, Najm IM: Indications and selection criteria for invasive monitoring in children with cortical dysplasia. Childs Nerv Syst 30:1823-1829, 2014

21. Hall JA, Khoo HM: Robotic-assisted and image-guided MRI-compatible stereoelectroencephalography. Can J Neurol Sci 45:35-43, 2018

22. Hou Z, Chen X, Shi XJ, An N, Yang MH, Yang H, et al: Comparison of neuronavigation and frame-based stereotactic systems in implanting epileptic depth electrodes. Turk Neurosurg 26:574-581, 2016

23. Isnard J, Taussig D, Bartolomei F, Bourdillon P, Catenoix H, Chassoux F, et al: French guidelines on stereoelectroencephalography (SEEG). Neurophysiol Clin 48:5-13, 2018

24. Johnston JM Jr, Mangano FT, Ojemann JG, Park TS, Trevathan E, Smyth MD: Complications of invasive subdural electrode monitoring at St. Louis Children's Hospital, 19942005. J Neurosurg 105 (5 Suppl):343-347, 2006

25. Kim H, Lee C, Knowlton R, Rozzelle C, Blount JP: Safety and utility of supplemental depth electrodes for localizing the ictal onset zone in pediatric neocortical epilepsy. J Neurosurg Pediatr 8:49-56, 2011

26. Lee JH, Hwang YS, Shin JJ, Kim TH, Shin HS, Park SK: 
Surgical complications of epilepsy surgery procedures: experience of 179 procedures in a single institute. J Korean Neurosurg Soc 44:234-239, 2008

27. MacDougall KW, Burneo JG, McLachlan RS, Steven DA: Outcome of epilepsy surgery in patients investigated with subdural electrodes. Epilepsy Res 85:235-242, 2009

28. Mathon B, Clemenceau S, Hasboun D, Habert MO, Belaid $\mathrm{H}$, Nguyen-Michel VH, et al: Safety profile of intracranial electrode implantation for video-EEG recordings in drugresistant focal epilepsy. J Neurol 262:2699-2712, 2015

29. McGonigal A, Bartolomei F, Régis J, Guye M, Gavaret M, Trébuchon-Da Fonseca A, et al: Stereoelectroencephalography in presurgical assessment of MRI-negative epilepsy. Brain 130:3169-3183, 2007

30. Mehta AD, Labar D, Dean A, Harden C, Hosain S, Pak J, et al: Frameless stereotactic placement of depth electrodes in epilepsy surgery. J Neurosurg 102:1040-1045, 2005

31. Miller BA, Salehi A, Limbrick DD Jr, Smyth MD: Applications of a robotic stereotactic arm for pediatric epilepsy and neurooncology surgery. J Neurosurg Pediatr 20:364-370, 2017

32. Minotti L, Montavont A, Scholly J, Tyvaert L, Taussig D: Indications and limits of stereoelectroencephalography (SEEG). Neurophysiol Clin 48:15-24, 2018

33. Mullin JP, Sexton D, Al-Omar S, Bingaman W, GonzalezMartinez J: Outcomes of subdural grid electrode monitoring in the stereoelectroencephalography era. World Neurosurg 89:255-258, 2016

34. Murphy MA, O'Brien TJ, Cook MJ: Insertion of depth electrodes with or without subdural grids using frameless stereotactic guidance systems - technique and outcome. Br J Neurosurg 16:119-125, 2002

35. Musleh W, Yassari R, Hecox K, Kohrman M, Chico M, Frim $\mathrm{D}$ : Low incidence of subdural grid-related complications in prolonged pediatric EEG monitoring. Pediatr Neurosurg 42:284-287, 2006

36. Nowell M, Rodionov R, Diehl B, Wehner T, Zombori G, Kinghorn J, et al: A novel method for implementation of frameless StereoEEG in epilepsy surgery. Neurosurgery 10 (Suppl 4):525-534, 2014

37. Önal C, Otsubo H, Araki T, Chitoku S, Ochi A, Weiss S, et al: Complications of invasive subdural grid monitoring in children with epilepsy. J Neurosurg 98:1017-1026, 2003

38. Roessler K, Sommer B, Merkel A, Rampp S, Gollwitzer S, Hamer HM, et al: A frameless stereotactic implantation technique for depth electrodes in refractory epilepsy using intraoperative magnetic resonance imaging. World Neurosurg 94:206-210, 2016

39. Serletis D, Bulacio J, Bingaman W, Najm I, GonzálezMartínez J: The stereotactic approach for mapping epileptic networks: a prospective study of 200 patients. J Neurosurg 121:1239-1246, 2014

40. Simon SL, Telfeian A, Duhaime AC: Complications of invasive monitoring used in intractable pediatric epilepsy. Pediatr Neurosurg 38:47-52, 2003

41. Spetzger U, Laborde G, Gilsbach JM: Frameless neuronavigation in modern neurosurgery. Minim Invasive Neurosurg 38:163-166, 1995

42. Spyrantis A, Cattani A, Strzelczyk A, Rosenow F, Seifert V, Freiman TM: Robot-guided stereoelectroencephalography without a computed tomography scan for referencing: analysis of accuracy. Int J Med Robot 14:e1888, 2018

43. Talairach J, Bancaud J, Szikla G, Bonis A, Geier S, Vedrenne $\mathrm{C}$ : [New approach to the neurosurgery of epilepsy. Stereo- taxic methodology and therapeutic results. 1. Introduction and history.] Neurochirurgie 20 (Suppl 1):1-240, 1974 (Fr)

44. Tanriverdi T, Ajlan A, Poulin N, Olivier A: Morbidity in epilepsy surgery: an experience based on 2449 epilepsy surgery procedures from a single institution. J Neurosurg 110:11111123,2009

45. Taussig D, Chipaux M, Lebas A, Fohlen M, Bulteau C, Ternier J, et al: Stereo-electroencephalography (SEEG) in 65 children: an effective and safe diagnostic method for pre-surgical diagnosis, independent of age. Epileptic Disord 16:280-295, 2014

46. Vadera S, Mullin J, Bulacio J, Najm I, Bingaman W, Gonzalez-Martinez J: Stereoelectroencephalography following subdural grid placement for difficult to localize epilepsy. Neurosurgery 72:723-729, 2013

47. Vakharia VN, Sparks R, O'Keeffe AG, Rodionov R, Miserocchi A, McEvoy A, et al: Accuracy of intracranial electrode placement for stereoencephalography: a systematic review and meta-analysis. Epilepsia 58:921-932, 2017

48. van der Loo LE, Schijns OEMG, Hoogland G, Colon AJ, Wagner GL, Dings JTA, et al: Methodology, outcome, safety and in vivo accuracy in traditional frame-based stereoelectroencephalography. Acta Neurochir (Wien) 159:1733-1746, 2017

49. Van Gompel JJ, Worrell GA, Bell ML, Patrick TA, Cascino GD, Raffel C, et al: Intracranial electroencephalography with subdural grid electrodes: techniques, complications, and outcomes. Neurosurgery 63:498-506, 2008

50. Verburg N, Baayen JC, Idema S, Klitsie MAJ, Claus S, de Jonge CS, et al: In vivo accuracy of a frameless stereotactic drilling technique for diagnostic biopsies and stereoelectroencephalography depth electrodes. World Neurosurg 87:392-398, 2016

51. Wong CH, Birkett J, Byth K, Dexter M, Somerville E, Gill $\mathrm{D}$, et al: Risk factors for complications during intracranial electrode recording in presurgical evaluation of drug resistant partial epilepsy. Acta Neurochir (Wien) 151:37-50, 2009

52. Yang M, Ma Y, Li W, Shi X, Hou Z, An N, et al: A retrospective analysis of stereoelectroencephalography and subdural electroencephalography for preoperative evaluation of intractable epilepsy. Stereotact Funct Neurosurg 95:13-20, 2017

53. Zhang G, Chen G, Meng D, Liu Y, Chen J, Shu L, et al: Stereoelectroencephalography based on the Leksell stereotactic frame and Neurotech operation planning software. Medicine (Baltimore) 96:e7106, 2017

\section{Disclosures}

The authors report no conflict of interest concerning the materials or methods used in this study or the findings specified in this paper.

\section{Author Contributions}

Conception and design: Grant, Ho. Acquisition of data: Ho, Feng, Kim. Analysis and interpretation of data: Grant, Ho, Feng, Kim. Drafting the article: Ho, Feng, Kim. Critically revising the article: Grant, Ho, Pendharkar, Sussman, Halpern. Reviewed submitted version of manuscript: all authors. Administrative/technical/material support: Grant, Halpern. Study supervision: Grant, Halpern.

\section{Correspondence}

Gerald A. Grant: Stanford University/Lucile Packard Children's Hospital, Stanford, CA. ggrant2@stanford.edu. 